Gut and Liver, Vol. 11, No. 1, January 2017, pp. 9-10

\title{
Another Face of Contrast-Enhanced Ultrasonography: A Diagnostic Tool for Cirrhosis
}

\author{
Soung Won Jeong \\ Department of Internal Medicine, Soonchunhyang University Seoul Hospital, Soonchunhyang University College of Medicine, Seoul, Korea
}

See "Diagnostic Accuracy of Hepatic Vein Arrival Time Performed with Contrast-Enhanced Ultrasonography for Cirrhosis: A Systematic Review and Meta-Analysis" by Gaeun Kim, et al. on page 93, Vol. 11. No. 1, 2017

Contrast-enhanced ultrasonography (CEUS) is a useful noninvasive diagnostic tool allowing accurate differentiation between benign and malignant liver tumors and the diagnosis of small, new liver nodules during surveillance for hepatocellular carcinoma (HCC). ${ }^{1}$ CEUS also can be applied to guide and assess locoregional HCC therapy., ${ }^{2,3}$ Low mechanical index ultrasound in combination with ultrasound contrast agent allows the real-time assessment of tumor vascularity and enhancement throughout the different vascular phases including arterial, portal venous, and delayed phases. ${ }^{4}$ However, CEUS has another useful function to detect the change of blood flow. When liver fibrosis progresses to cirrhosis, this is accompanied by intrahepatic hemodynamic changes including liver arterialization, and intrahepatic and pulmonary arteriovenous shunting. Although Doppler ultrasound is the first-line imaging tool for blood flow, this cannot be used to analyze flow in capillaries and sinusoids; the flow is too slow to produce a Doppler signal. ${ }^{5}$ However, the microbubbles of CEUS acts as a blood pool tracer and enables arrival and transit time measurements and can analyze the change of blood flow in capillaries and sinusoids of cirrhosis. The hepatic vein arrival time (HVAT) is the time taken, commencing at injection, for the microbubble contrast agent to arrive at the hepatic vein. ${ }^{6}$ Although liver biopsy is the gold standard for diagnosis of fibrosis, the procedure is invasive and sampling errors might affect the accuracy of the results. Therefore, there is a great need to find a non-invasive method for the assessment of hepatic fibrosis. Recently, several authors have used CEUS to diagnose cirrhosis. In the present issue of Gut and Liver, Kim et al. ${ }^{7}$ investigated the HVAT measured by CEUS to assess hepatic fibrosis in cirrhosis based on a systematic review (SR) and meta- analysis (MA). The authors identified that the measurement of HVAT by CEUS exhibited an increased accuracy and correlation for the detection of cirrhosis. The HVAT of cirrhotic patients was shorter than that of noncirrhotic patients; this reduction was accompanied by an increase in the severity of liver disease attributable to intrahepatic hemodynamic changes. These reflect capillarization of the sinusoids or the arteriovenous or portovenous shunts. ${ }^{8,9}$ Many studies have reported the relationship between HVAT and the severity of liver histological grade. However, the reported accuracy and usefulness have been inconsistent across studies. In their SR and MA, the cited authors evaluated 12 studies on a total of 844 patients with (principally) hepatitis $\mathrm{B}$ or $\mathrm{C}$ virus infections. All were case control studies comparing patients with biopsy-confirmed cirrhosis to control groups. In terms of the contrast agent, five studies used Levovist (Schering AG) and six SonoVue (Bracco SpA). Sonazoid (GE Healthcare) and Definity (Lantheus Medical Imaging) were each used in one study. The HVAT was measured using the Doppler test in nine of 12 studies, and the remaining three studies were assessed by visual inspection. The diagnostic accuracy of CEUS was high. The integrated sensitivity was 0.83 (95\% confidence interval [CI], 0.77 to 0.89 ), the specificity 0.75 (95\% CI, 0.69 to 0.79 ), and the summary receiver operating characteristic area under the curve 0.74 (standard error, 0.14). However, study heterogeneity was also high.

There are several issues to comment regarding this SR and MA. First, the results varied with the contrast agent used and the method employed to measure HVAT. As the HVAT is influenced by the microbubble extraction rate due to phagocytosis, Sonazoid with higher extraction rate by Kupffer cells showed

Correspondence to: Soung Won Jeong

Department of Internal Medicine, Soonchunhyang University Seoul Hospital, Soonchunhyang University College of Medicine, 59 Daesagwan-ro, Yongsan-gu, Seoul 04401, Korea

Tel: +82-2-710-3076, Fax: +82-2-709-9696, E-mail: jeongsw@schmc.ac.kr pISSN 1976-2283 eISSN 2005-1212 https://doi.org/10.5009/gnl16563

@ This is an Open Access article distributed under the terms of the Creative Commons Attribution Non-Commercial License (http://creativecommons.org/licenses/by-nc/4.0) which permits unrestricted non-commercial use, distribution, and reproduction in any medium, provided the original work is properly cited. 
lower diagnostic accuracies compared to Levovist and SonoVue that undergoes less phagocytosis and remains mainly in the blood vessels. In terms of HVAT calculation, visual inspection was associated with a lower diagnostic accuracy than US/Doppler evaluation. Finally, a study using SonoVue as the contrast agent and US/Doppler to calculate the HVAT afforded high sensitivity (1.0) and specificity (0.93). Secondly, this SR and MA evaluated only 12 studies and the characteristics of each study was various according to patient characteristics, etiology of cirrhosis, contrast agents, and the calculating method of HVAT. Indeed, the unified cutoff value of HVAT for diagnosing the fibrosis and cirrhosis is required using the effective contrast agent and HVAT measurement such as Sonovue combined with US/ Doppler. Future large number of randomized controlled studies with homogenous patient characteristics and methodology is needed. Thirdly, there have been many diagnostic approaches for liver fibrosis including invasive methods with liver biopsy, hepatic venous pressure gradient, and nonivasive approach with transient elastography (FibroScan), magnetic resonance imaging, magnetic resonance spectroscopy, and serum markers. Recently, Kim et al. ${ }^{7}$ prospectively evaluated the relationship between hepatic venous pressure gradient and HVAT and there was a significant negative correlation, suggesting that HVAT is useful for the noninvasive prediction of clinically significant portal hypertension in patients with compensated cirrhosis. ${ }^{10}$ However, there are few studies to compare CEUS with other noninvasive diagnostic tests including FibroScan. CEUS is minimally invasive and uses shunt-related diagnostic approach based on intra and extrahepatic hemodynamic changes. It is essential to find the suitable position of CEUS in the diagnosis of cirrhosis.

\section{CONFLICTS OF INTEREST}

No potential conflict of interest relevant to this article was reported.

\section{ACKNOWLEDGEMENTS}

This study was supported by the Soonchunhyang University Research Fund.

\section{REFERENCES}

1. Ryu SW, Bok GH, Jang JY, et al. Clinically useful diagnostic tool of contrast enhanced ultrasonography for focal liver masses: comparison to computed tomography and magnetic resonance imaging. Gut Liver 2014;8:292-297.

2. Park KH, Kwon SH, Lee YS, et al. Predictive factors of contrastenhanced ultrasonography for the response to transarterial chemoembolization in hepatocellular carcinoma. Clin Mol Hepatol 2015;21:158-164.

3. Minami T, Minami $\mathrm{Y}$, Chishina $\mathrm{H}$, et al. Combination guidance of contrast-enhanced US and fusion imaging in radiofrequency ablation for hepatocellular carcinoma with poor conspicuity on contrast-enhanced US/fusion imaging. Oncology 2014;87 Suppl 1:55-62.

4. Nicolau C, Vilana R, Catalá V, et al. Importance of evaluating all vascular phases on contrast-enhanced sonography in the differentiation of benign from malignant focal liver lesions. AJR Am J Roentgenol 2006;186:158-167.

5. Desser TS, Sze DY, Jeffrey RB. Imaging and intervention in the hepatic veins. AJR Am J Roentgenol 2003;180:1583-1591.

6. Albrecht T, Blomley MJ, Cosgrove DO, et al. Non-invasive diagnosis of hepatic cirrhosis by transit-time analysis of an ultrasound contrast agent. Lancet 1999;353:1579-1583.

7. Kim G, Shim KY, Baik SK. Diagnostic accuracy of hepatic vein arrival time performed with contrast-enhanced ultrasonography for cirrhosis: a systematic review and meta-analysis. Gut Liver 2017;11:93-101.

8. Baik SK. Haemodynamic evaluation by Doppler ultrasonography in patients with portal hypertension: a review. Liver Int 2010;30:1403-1413.

9. Grier S, Lim AK, Patel N, et al. Role of microbubble ultrasound contrast agents in the non-invasive assessment of chronic hepatitis C-related liver disease. World J Gastroenterol 2006;12:34613465.

10. Kim MY, Suk KT, Baik SK, et al. Hepatic vein arrival time as assessed by contrast-enhanced ultrasonography is useful for the assessment of portal hypertension in compensated cirrhosis. Hepatology 2012;56:1053-1062. 